\title{
NIELSEN FIXED POINT THEORY AND SYMPLECTIC FLOER HOMOLOGY
}

\author{
ALEXANDER FEL'SHTYN \\ Instytut Matematyki, Uniwersytet Szczecinski \\ ul. Wielkopolska 15, 70-451 Szczecin, Poland \\ and \\ Boise State University \\ 1910 University Drive, Boise, Idaho, 83725-155, USA \\ E-mail: felshtyn@diamond.boisestate.edu,felshtyn@mpim-bonn.mpg.de
}

\begin{abstract}
We describe a connection between Nielsen fixed point theory and symplectic Floer homology for surfaces. A new asymptotic invariant of symplectic origin is defined.
\end{abstract}

1. Introduction. In the dimension two a diffeomorphism is symplectic if it preserves the area. As a consequence, the symplectic geometry of surfaces lacks many interesting phenomena which are encountered in higher dimensions. For example, two symplectic automorphisms of a closed surface are symplectically isotopic iff they are homotopic, by a theorem of Moser[19]. On the other hand, symplectic fixed point theory is very nontrivial in dimension 2, as it is shown by the Poincaré-Birkhoff theorem. It is known that symplectic Floer homology on surface is a simple model for the instanton Floer homology of the mapping torus of the surface diffeomorphism [21].

In this article we prove the following result: Let $M$ be a compact connected surface of Euler characteristic $\chi(M)<0$. If $\phi$ is a non-trivial orientation preserving periodic diffeomorphism of $M$ or $\phi$ is a diffeomorphism of finite type with only isolated fixed points, then $\phi$ is monotone with respect to some $\phi$-invariant area form on $M$ and $H F_{*}(\phi) \cong$ $\mathbb{Z}_{2}^{N(\phi)}, \operatorname{dim} H F_{*}(\phi)=N(\phi)$, where $N(\phi)$ denotes the Nielsen number of $\phi$ and $H F_{*}(\phi)$ denotes symplectic Floer homology group.

We also define asymptotic invariant of monotone symplectomorphism.

The author came to the idea that Nielsen numbers are connected with Floer homology of surface diffeomorphisms at the Autumn 2000, after conversations with Joel Robbin and Dan Burghelea. I am very grateful to Dietmar Salamon who sent me handwritten notes of Wu-Chung Hsiang paper A speculation on Floer theory and Nielsen theory and to

2000 Mathematics Subject Classification: 37C25, 53D, 37C30, 55M20.

The paper is in final form and no version of it will be published elsewhere. 
Wu-Chung Hsiang, Jarek Kędra, François Laudenbach, Kaoru Ono, Yuli Rudyak, Andrei Tyurin and Vladimir Turaev for very useful discussions of symplectic Floer homology.

\section{Symplectic Floer homology}

2.1. Monotonicity. In this section we discuss the notion of monotonicity as defined in $[21,8]$. Monotonicity plays important role for Floer homology in two dimensions. For a more detailed account we refer to the original articles of P. Seidel and R. Gautschi.

Throughout this article, $M$ denotes a closed connected and oriented 2-manifold of genus $\geq 2$. Pick an everywhere positive two-form $\omega$ on $M$.

Let $\operatorname{Symp}(M, \omega)$ denote the group of symplectic automorphisms of the two-dimensional symplectic manifold $(M, \omega)$ and $\phi \in \operatorname{Symp}(M, \omega)$. The mapping torus of $\phi, T_{\phi}=$ $\mathbb{R} \times M /(t+1, x) \sim(t, \phi(x))$, is a 3-manifold fibered over $S^{1}=\mathbb{R} / \mathbb{Z}$. There are two natural second cohomology classes on $T_{\phi}$, denoted by $\left[\omega_{\phi}\right]$ and $c_{\phi}$. The first one is represented by the closed two-form $\omega_{\phi}$ which is induced from the pullback of $\omega$ to $\mathbb{R} \times M$. The second is the Euler class of the vector bundle

$$
V_{\phi} \mathbb{R} \times T M /\left(t+1, \xi_{x}\right) \sim\left(t, \mathrm{~d} \phi_{x} \xi_{x}\right),
$$

which is of rank 2 and inherits an orientation from $T M$.

A symplectomorphism $\phi \in \operatorname{Symp}(M, \omega)$ is called monotone, if

$$
\left[\omega_{\phi}\right]=\left(\operatorname{area}_{\omega}(M) / \chi(M)\right) \cdot c_{\phi}
$$

in $H^{2}\left(T_{\phi} ; \mathbb{R}\right)$; throughout this article $\operatorname{Symp}^{m}(M, \omega)$ denotes the set of monotone symplectomorphisms.

Now $H^{2}\left(T_{\phi} ; \mathbb{R}\right)$ fits into the following short exact sequence $[21,8]$

$$
0 \longrightarrow \frac{H^{1}(M ; \mathbb{R})}{\operatorname{im}\left(\mathrm{id}-\phi^{*}\right)} \stackrel{d}{\longrightarrow} H^{2}\left(T_{\phi} ; \mathbb{R}\right) \stackrel{r^{*}}{\longrightarrow} H^{2}(M ; \mathbb{R}) \longrightarrow 0 .
$$

where the map $r^{*}$ is restriction to the fiber. The map $d$ is defined as follows. Let $\rho: I \rightarrow \mathbb{R}$ be a smooth function which vanishes near 0 and 1 and satisfies $\operatorname{int}_{0}^{1} \rho d t=1$. If $\theta$ is a closed 1-form on $M$, then $\rho \cdot \theta \wedge d t$ defines a closed 2-form on $T_{\phi}$; indeed $d[\theta]=[\rho \cdot \theta \wedge d t]$. The map $r: M \hookrightarrow T_{\phi}$ assigns to each $x \in M$ the equivalence class of $(1 / 2, x)$. Note that $r^{*} \omega_{\phi}=\omega$ and $r^{*} c_{\phi}$ is the Euler class of $T M$. Hence, by (1), there exists a unique class $m(\phi) \in H^{1}(M ; \mathbb{R}) / \operatorname{im}\left(\mathrm{id}-\phi^{*}\right)$ satisfying

$$
d m(\phi)=\left[\omega_{\phi}\right]-\left(\operatorname{area}_{\omega}(M) / \chi(M)\right) \cdot c_{\phi},
$$

where $\chi$ denotes the Euler characteristic. Therefore, $\phi$ is monotone if and only if $m(\phi)=0$.

We recall the fundamental properties of $\operatorname{Symp}^{m}(M, \omega)$ from $[21,8]$. Let $\operatorname{Diff}^{+}(M)$ denote the group of orientation preserving diffeomorphisms of $M$.

(Identity) $\operatorname{id}_{M} \in \operatorname{Symp}^{m}(M, \omega)$.

(Naturality) If $\phi \in \operatorname{Symp}^{m}(M, \omega), \psi \in \operatorname{Diff}^{+}(M)$, then $\psi^{-1} \phi \psi \in \operatorname{Symp}^{m}\left(M, \psi^{*} \omega\right)$.

(Isotopy) Let $\left(\psi_{t}\right)_{t \in I}$ be an isotopy in $\operatorname{Symp}(M, \omega)$, i.e. a smooth path with $\psi_{0}=$ id. Then $m\left(\phi \circ \psi_{1}\right)=m(\phi)+\left[\operatorname{Flux}\left(\psi_{t}\right)_{t \in I}\right]$ in $H^{1}(M ; \mathbb{R}) / \operatorname{im}\left(\mathrm{id}-\phi^{*}\right)$; see $[21$, Lemma 6]. For the definition of the flux homomorphism see [17]. 
(Inclusion) The inclusion $\operatorname{Symp}^{m}(M, \omega) \hookrightarrow \operatorname{Diff}^{+}(M)$ is a homotopy equivalence. This follows from the isotopy property, surjectivity of the flux homomorphism and Moser's isotopy theorem [19], which says that each element of the mapping class group admits representatives which preserve $\omega$. Furthermore, the Earl-Eells Theorem [3] implies that every connected component of $\operatorname{Symp}^{m}(M, \omega)$ is contractible.

(Floer homology) The symplectic Floer homology theory assigns a $\mathbb{Z}_{2}$-graded vector space $H F_{*}(\phi)$ over $\mathbb{Z}_{2}$ to every $\phi \in \operatorname{Symp}^{m}(M, \omega)$. Furthermore, $H F_{*}(\phi)$ possesses an additional multiplicative structure, called the quantum cap product, $H^{*}\left(M ; \mathbb{Z}_{2}\right) \otimes$ $H F_{*}(\phi) \longrightarrow H F_{*}(\phi)$. For $\phi=\mathrm{id}_{M}$ the symplectic Floer homology $H F_{*}\left(\operatorname{id}_{M}\right)$ are canonically isomorphic to ordinary homology $H_{*}\left(M ; \mathbb{Z}_{2}\right)$ and quantum cap product agrees with the ordinary cap product. Each $\psi \in \operatorname{Diff}^{+}(M)$ induces an isomorphism $H F_{*}(\phi) \cong$ $H F_{*}\left(\psi^{-1} \phi \psi\right)$ of $H^{*}\left(M ; \mathbb{Z}_{2}\right)$-modules.

(Invariance) If $\phi, \phi^{\prime} \in \operatorname{Symp}^{m}(M, \omega)$ are isotopic, then $H F_{*}(\phi)$ and $H F_{*}\left(\phi^{\prime}\right)$ are naturally isomorphic as $H^{*}\left(M ; \mathbb{Z}_{2}\right)$-modules. This is proven in [21, p. 7]. Note that every Hamiltonian perturbation of $\phi$ (see [2]) is also in $\operatorname{Symp}^{m}(M, \omega)$.

Now, let $g$ be a mapping class of $M$, i.e. an isotopy class of $\operatorname{Diff}^{+}(M)$. Pick an area form $\omega$ and a representative $\phi \in \operatorname{Symp}^{m}(M, \omega)$ of $g$. Then $H F_{*}(\phi)$ is an invariant of $g$, which is denoted by $H F_{*}(g)$. Note that $H F_{*}(g)$ is independent of the choice of an area form $\omega$ by Moser's isotopy theorem [19] and naturality of Floer homology.

2.2. Floer homology. Let $\phi \in \operatorname{Symp}(M, \omega)$. There are two ways of constructing Floer homology detecting its fixed points, $\operatorname{Fix}(\phi)$. Firstly, the graph of $\phi$ is a Lagrangian submanifold of $(M \times M,(-\omega) \times \omega)$ and its fixed points correspond to the intersection points of $\operatorname{graph}(\phi)$ with the diagonal $\Delta=\{(x, x) \in M \times M\}$. Thus we have the Floer homology of the Lagrangian intersection $H F_{*}(M \times M, \Delta$, graph $(\phi))$. This intersection is transversal if the fixed points of $\phi$ are non-degenerate, i.e. if 1 is not an eigenvalue of $d \phi(x)$, for $x \in \operatorname{Fix}(\phi)$. The second approach was mentioned by Floer in [6] and presented with details by Dostoglou and Salamon in [2]. We follow here Seidel's approach [21] which, comparable with [2], uses a larger class of perturbations, but such that the perturbed action form is still cohomologous to the unperturbed. As a consequence, the usual invariance of Floer homology under Hamiltonian isotopies is extended to the stronger property stated above. Let now $\phi \in \operatorname{Symp}^{m}(M, \omega)$, i.e., $\phi$ be monotone. Firstly, we give the definition of $H F_{*}(\phi)$ in the special case where all the fixed points of $\phi$ are non-degenerate, i.e. for all $y \in \operatorname{Fix}(\phi)$, $\operatorname{det}\left(\mathrm{id}-\mathrm{d} \phi_{y}\right) \neq 0$, and then following Seidel's approach [21] we consider general case when $\phi$ has degenerate fixed points. Let $\Omega_{\phi}=\left\{y \in C^{\infty}(\mathbb{R}, M): y(t)=\phi(y(t+1))\right\}$ be the twisted free loop space, which is also the space of sections of $T_{\phi} \rightarrow S^{1}$. The action form is the closed one-form $\alpha_{\phi}$ on $\Omega_{\phi}$ defined by

$$
\alpha_{\phi}(y) Y=\operatorname{int}_{0}^{1} \omega(d y / d t, Y(t)) d t .
$$

where $y \in \Omega_{\phi}$ and $Y \in T_{y} \Omega_{\phi}$, i.e. $Y(t) \in T_{y(t)} M$ and $Y(t)=\mathrm{d} \phi_{y(t+1)} Y(t+1)$ for all $t \in \mathbb{R}$.

The tangent bundle of any symplectic manifold admits an almost complex structure $J: T M \longrightarrow T M$ which is compatible with $\omega$ in the sense that $(v, w)=\omega(v, J w)$ defines 
a Riemannian metric. Let $J=\left(J_{t}\right)_{t \in \mathbb{R}}$ be a smooth path of $\omega$-compatible almost complex structures on $M$ such that $J_{t+1}=\phi^{*} J_{t}$. If $Y, Y^{\prime} \in T_{y} \Omega_{\phi}$, then $\operatorname{int}_{0}^{1} \omega\left(Y^{\prime}(t), J_{t} Y(t)\right) d t$ defines a metric on the loop space $\Omega_{\phi}$. So the critical points of $\alpha_{\omega}$ are the constant paths in $\Omega_{\phi}$ and hence the fixed points of $\phi$. The negative gradient lines of $\alpha_{\omega}$ with respect to the metric above are solutions of the partial differential equations with boundary conditions

$$
\left\{\begin{array}{l}
u(s, t)=\phi(u(s, t+1)), \\
\partial_{s} u+J_{t}(u) \partial_{t} u=0, \\
\lim _{s \rightarrow \pm \infty} u(s, t) \in \operatorname{Fix}(\phi)
\end{array}\right.
$$

These are exactly Gromov's pseudoholomorphic curves [10].

For $y^{ \pm} \in \operatorname{Fix}(\phi)$, let $\mathcal{M}\left(y^{-}, y^{+} ; J, \phi\right)$ denote the space of smooth maps $u: \mathbb{R}^{2} \rightarrow M$ which satisfy the equations (2). Now to every $u \in \mathcal{M}\left(y^{-}, y^{+} ; J, \phi\right)$ we associate a Fredholm operator $\mathrm{D}_{u}$ which linearizes $(2)$ in suitable Sobolev spaces. The index of this operator is given by the so-called Maslov index $\mu(u)$, which satisfies $\mu(u)=\operatorname{deg}\left(y^{+}\right)-\operatorname{deg}\left(y^{-}\right) \bmod 2$, where $(-1)^{\operatorname{deg} y}=\operatorname{sign}\left(\operatorname{det}\left(\mathrm{id}-\mathrm{d} \phi_{y}\right)\right)$. We have no bubbling, since for surface $\pi_{2}(M)=0$. For a generic $J$, every $u \in \mathcal{M}\left(y^{-}, y^{+} ; J, \phi\right)$ is regular, meaning that $\mathrm{D}_{u}$ is onto. Hence, by the implicit function theorem, $\mathcal{M}_{k}\left(y^{-}, y^{+} ; J, \phi\right)$ is a smooth $k$-dimensional manifold, where $\mathcal{M}_{k}\left(y^{-}, y^{+} ; J, \phi\right)$ denotes the subset of those $u \in \mathcal{M}\left(y^{-}, y^{+} ; J, \phi\right)$ with $\mu(u)=k \in \mathbb{Z}$. Translation of the $s$-variable defines a free $\mathbb{R}$-action on 1-dimensional manifold $\mathcal{M}_{1}\left(y^{-}, y^{+} ; J, \phi\right)$ and hence the quotient is a discrete set of points. The energy of a map $u: \mathbb{R}^{2} \rightarrow M$ is given by $E(u)=\operatorname{int}_{\mathbb{R}} \operatorname{int}_{0}^{1} \omega\left(\partial_{t} u(s, t), J_{t} \partial_{t} u(s, t)\right) d t d s$ for all $y \in \operatorname{Fix}(\phi)$. P. Seidel has proved in [21] that if $\phi$ is monotone, then the energy is constant on each $\mathcal{M}_{k}\left(y^{-}, y^{+} ; J, \phi\right)$. Since all fixed points of $\phi$ are non-degenerate, the set $\operatorname{Fix}(\phi)$ is a finite set and the $\mathbb{Z}_{2}$-vector space $C F_{*}(\phi):=\mathbb{Z}_{2}^{\# \operatorname{Fix}(\phi)}$ admits a $\mathbb{Z}_{2}$-grading with $(-1)^{\operatorname{deg} y}=\operatorname{sign}\left(\operatorname{det}\left(\mathrm{id}-\mathrm{d} \phi_{y}\right)\right)$, for all $y \in \operatorname{Fix}(\phi)$. The boundness of the energy $E(u)$ for monotone $\phi$ implies that the 0 -dimensional quotients $\mathcal{M}_{1}\left(y_{-}, y_{+}, J, \phi\right) / \mathbb{R}$ are actually finite sets. Denoting by $n\left(y_{-}, y_{+}\right)$the number of points $\bmod 2$ in each of them, one defines a differential $\partial_{J}: C F_{*}(\phi) \rightarrow C F_{*+1}(\phi)$ by $\partial_{J} y_{-} \sum_{y_{+}} n\left(y_{-}, y_{+}\right) y_{+}$. Due to gluing theorem this Floer boundary operator satisfies $\partial_{J} \circ \partial_{J}=0$. For gluing theorem to hold one needs again the boundness of the energy $E(u)$. It follows that $\left(C F_{*}(\phi), \partial_{J}\right)$ is a chain complex and its homology is by definition the Floer homology of $\phi$ denoted by $H F_{*}(\phi)$. It is independent of $J$ and is an invariant of $\phi$.

If $\phi$ has degenerate fixed points one needs to perturb equations (2) in order to define the Floer homology. Equivalently, one could say that the action form needs to be perturbed. The necessary analysis is given in [21], it is essentially the same as in the slightly different situations considered in [2]. But Seidel's approach also differs from the usual one in [2]. He uses a larger class of perturbations, but such that the perturbed action form is still cohomologous to the unperturbed.

3. Nielsen numbers and Floer homology. Before discussing the main results of the paper, we briefly describe the few basic notions of Nielsen fixed point theory which will be used. We assume $X$ to be a connected, compact polyhedron and $f: X \rightarrow X$ to be a continuous map. Let $p: \tilde{X} \rightarrow X$ be the universal cover of $X$ and $\tilde{f}: \tilde{X} \rightarrow \tilde{X}$ a lifting of $f$, 
i.e. $p \circ \tilde{f}=f \circ p$. Two liftings $\tilde{f}$ and $\tilde{f}^{\prime}$ are called conjugate if there is a $\gamma \in \Gamma \cong \pi_{1}(X)$ such that $\tilde{f}^{\prime}=\gamma \circ \tilde{f} \circ \gamma^{-1}$. The subset $p(\operatorname{Fix}(\tilde{f})) \subset \operatorname{Fix}(f)$ is called the fixed point class of $f$ determined by the lifting class $[\tilde{f}]$. Two fixed points $x_{0}$ and $x_{1}$ of $f$ belong to the same fixed point class iff there is a path $c$ from $x_{0}$ to $x_{1}$ such that $c \cong f \circ c$ (homotopy relative endpoints). This fact can be considered as an equivalent definition of a non-empty fixed point class. Every map $f$ has only finitely many non-empty fixed point classes, each a compact subset of $X$. A fixed point class is called essential if its index is non-zero. The number of essential fixed point classes is called the Nielsen number of $f$, denoted by $N(f)$. The Nielsen number is always finite. $N(f)$ is a homotopy type invariant. In the category of compact, connected polyhedra, the Nielsen number of a map is, apart from certain exceptional cases, equal to the least number of fixed points of maps with the same homotopy type as $f$.

3.1. Periodic diffeomorphisms. The following lemma was first proven in [14]. We repeat here the arguments from [8].

LEMMA 1 ([14]). Let $\phi$ be a non-trivial orientation preserving periodic diffeomorphism of a compact connected surface $M$ of Euler characteristic $\chi(M) \leq 0$. Then each fixed point class of $\phi$ consists of a single point.

Proof. First assume that $M$ is closed. The uniformization theorem states that in every conformal class of metrics on $M$, there is a unique metric of constant curvature -1 if $\chi(M)<0$ or of constant curvature 0 if $\chi(M)=0$. This implies that the unique representative of a $\phi$-invariant conformal class of metrics (such a class exists since $\phi$ is of finite order) is $\phi$-invariant in itself. Hence we can pick a $\phi$-invariant metric of constant curvature -1 or 0 on $M$ and lift $\phi$ to an isometry $\tilde{\phi}$ of the universal cover $\tilde{M}$ of $M$. Here $\tilde{M}$ is either isometric to the hyperbolic plane $H^{2}$ or the Euclidean plane $\mathbb{R}^{2}$.

Let $x \in \operatorname{Fix}(\phi)$ and let $\tilde{\phi}, \tilde{x}$ be lifts of $\phi, x$ to $\tilde{M}$, such that $\tilde{\phi}(\tilde{x})=\tilde{x}$. Note that a fixed point of $\phi$ is in the same class as $x$ if and only if it can be lifted to a fixed point of $\tilde{\phi}$. Assume by contradiction that $\tilde{y} \neq \tilde{x}$ is a fixed point of $\tilde{\phi}$. It follows that the unique geodesic going through $\tilde{x}$ and $\tilde{y}$ is pointwise fixed by $\tilde{\phi}$. In particular, since $\tilde{\phi}$ preserves orientation, $\mathrm{d} \tilde{\phi}_{\tilde{x}}=\mathrm{id}$. This implies that $\tilde{\phi}=\mathrm{id}$, because an isometry of $H^{2}$ or $\mathbb{R}^{2}$ is determined by its value and differential at one point. This proves lemma in the case that $M$ is closed.

The case $\partial M \neq \emptyset$ is reduced to the above case by gluing two copies of $M$ together along a $\phi$-invariant tubular neighborhood of $\partial M$. The glued manifold is closed and of Euler characteristic $\leq 0 ; \varphi$ extends to a non-trivial diffeomorphism $\phi^{\prime}$, which is orientation preserving and of finite order. Hence, every fixed point class of $\phi^{\prime}$ is a single point. The same therefore holds for $\phi$. This completes the proof.

R. Gautschi gave two criteria for monotonicity which we use later on.

Let $\omega$ be an area form on $M$ and $\phi \in \operatorname{Symp}(\Sigma, \omega)$.

Lemma 2 ([8]). Assume that every class $\alpha \in \operatorname{ker}\left(\mathrm{id}-\phi_{*}\right) \subset H_{1}(M ; \mathbb{Z})$ is represented by a map $\gamma: S \rightarrow \operatorname{Fix}(\phi)$, where $S$ is a compact oriented 1-manifold. Then $\phi$ is monotone. 
LemMa 3 ([8]). If $\phi^{k}$ is monotone for some $k>0$, then $\phi$ is monotone. If $\phi$ is monotone, then $\phi^{k}$ is monotone for all $k>0$.

Proof. We repeat Gautschi's arguments from [8] here. Recall that $T_{\phi}$ is the orbit space of the $\mathbb{Z}$-action $n \cdot(t, x)=\left(t+n, \phi^{-n}(x)\right)$, where $n \in \mathbb{Z}$ and $(t, x) \in \mathbb{R} \times \Sigma$. If we divide out by the subgroup $k \mathbb{Z}$ only, $k \in \mathbb{N}_{>0}$, we naturally get the mapping torus of $\phi^{k}$. Further dividing by $\mathbb{Z} / k \mathbb{Z}$ defines the $k$-fold covering map $p_{k}: T_{\phi^{k}} \rightarrow T_{\phi}$. It is straightforward to check that

$$
p_{k}^{*}\left[\omega_{\phi}\right]=\left[\omega_{\phi^{k}}\right] \text { and } p_{k}^{*} c_{\phi}=c_{\phi^{k}} .
$$

The first equality follows immediately from the definitions. To prove the second, note that $p_{k}^{*}((T M \times \mathbb{R}) / \mathbb{Z}) \cong(T M \times \mathbb{R}) / k \mathbb{Z} \cong V_{\phi^{k}}$, where the $\mathbb{Z}$-action on $\mathbb{R} \times T \Sigma$ is given by $n \cdot\left(t, \xi_{x}\right)=\left(t+n, \mathrm{~d} \phi_{x}^{-n} \xi_{x}\right)$, for $n \in \mathbb{Z}$ and $\xi_{x} \in T_{x} M$. The lemma follows from (3) and the fact that $p_{k}^{*}$ is injective. To prove injectivity, define the map $a_{*}^{k}: H^{2}\left(T_{\phi^{k}} ; \mathbb{R}\right) \rightarrow H^{2}\left(T_{\phi} ; \mathbb{R}\right)$ by averaging differential forms; $a_{*}^{k}$ is a left inverse of $p_{k}^{*}$, i.e. $a_{*}^{k} \circ p_{k}^{*}=\mathrm{id}$. This ends the proof of the lemma.

We shall say that $\phi: M \rightarrow M$ is a periodic map of period $m$, if $\phi^{m}$ is the identity $\operatorname{map}_{M}: M \rightarrow M$.

THEOREM 4. If $\phi$ is a non-trivial orientation preserving periodic diffeomorphism of a compact connected surface $M$ of Euler characteristic $\chi(M)<0$, then $\phi$ is monotone with respect to some $\phi$-invariant area form and

$$
H F_{*}(\phi) \cong \mathbb{Z}_{2}^{N(\phi)}, \quad \operatorname{dim} H F_{*}(\phi)=N(\phi),
$$

where $N(\phi)$ denotes the Nielsen number of $\phi$.

Proof. Let $\phi$ be a periodic diffeomorphism of least period $l$. First note that if $\tilde{\omega}$ is an area form on $M$, then area form $\omega:=\sum_{i=1}^{\ell}\left(\phi^{i}\right)^{*} \tilde{\omega}$ is $\phi$-invariant, i.e. $\phi \in \operatorname{Symp}(M, \omega)$. By periodicity of $\phi, \phi^{l}$ is the identity map $\operatorname{id}_{M}: M \rightarrow M$. Then from Lemmas 2 and 3 it follows that $\omega$ can be chosen such that $\phi \in \operatorname{Symp}^{m}(M, \omega)$.

Lemma 1 implies that every $y \in \operatorname{Fix}(\phi)$ forms a different fixed point class of $\phi$, so $\# \operatorname{Fix}(\phi)=N(\phi)$. This has an immediate consequence for the Floer complex $\left(C F_{*}(\phi), \partial_{J}\right)$ with respect to a generic $J=\left(J_{t}\right)_{t \in \mathbb{R}}$. If $y^{ \pm} \in \operatorname{Fix}(\phi)$ are in different fixed point classes, then $\mathcal{M}\left(y^{-}, y^{+} ; J, \phi\right)=\emptyset$. This follows from the first equation in (2). Then the boundary map in the Floer complex is zero $\partial_{J}=0$ and $\mathbb{Z}_{2}$-vector space $C F_{*}(\phi):=\mathbb{Z}_{2}^{\# \operatorname{Fix}(\phi)}=$ $\mathbb{Z}_{2}^{N(\phi)}$. This immediately implies $H F_{*}(\phi) \cong \mathbb{Z}_{2}^{N(\phi)}$ and $\operatorname{dim} H F_{*}(\phi)=N(\phi)$.

3.2. Algebraically finite mapping classes. A mapping class of $M$ is called algebraically finite if it has no pseudo-Anosov components in the sense of Thurston's theory of surface diffeomorphism. The term "algebraically finite" goes back to J. Nielsen.

R. Gautschi [8] defined the notion of a diffeomorphism of finite type for surface diffeomorphisms. These are special representatives of algebraically finite mapping classes adopted to the symplectic geometry.

Definition 5 ([8]). We call $\phi \in \operatorname{Diff}_{+}(M)$ of finite type if the following holds. There is a $\phi$-invariant finite union $N \subset M$ of disjoint non-contractible annuli such that: 
(1) $\phi \mid M \backslash N$ is periodic, i.e. there exists $\ell>0$ such that $\phi^{\ell} \mid M \backslash N=$ id.

(2) Let $N^{\prime}$ be a connected component of $N$ and $\ell^{\prime}>0$ be the smallest integer such that $\phi^{\ell^{\prime}}$ maps $N^{\prime}$ to itself. Then $\phi^{\ell^{\prime}} \mid N^{\prime}$ is given by one of the following two models with respect to some coordinates $(q, p) \in I \times S^{1}$ :

$$
\begin{array}{lll}
\text { (twist map) } & (q, p) \longmapsto(q, p-f(q)) \\
\text { (flip-twist map) } & (q, p) \longmapsto(1-q,-p-f(q)),
\end{array}
$$

where $f: I \rightarrow \mathbb{R}$ is smooth and strictly monotone. A twist map is called positive or negative, if $f$ is increasing or decreasing.

(3) Let $N^{\prime}$ and $\ell^{\prime}$ be as in (2). If $\ell^{\prime}=1$ and $\phi \mid N^{\prime}$ is a twist map, then $\operatorname{im}(f) \subset[0,1]$, i.e. $\phi \mid \operatorname{int}\left(N^{\prime}\right)$ has no fixed points.

(4) If two connected components of $N$ are homotopic, then the corresponding local models of $\phi$ are either both positive or both negative twists.

The term "flip-twist map" is taken from [16].

By $M_{\text {id }}$ we denote the union of the components of $M \backslash \operatorname{int}(N)$, where $\phi$ restricts to the identity.

The next lemma describes the set of fixed point classes of $\phi$. It is a special case of a theorem by B. Jiang and J. Guo [16], which gives for any mapping class a representative that realizes its Nielsen number.

LEMma 6 (Fixed point classes [16]). Each fixed point class of $\phi$ either is a connected component of $M_{\mathrm{id}}$ or consists of a single fixed point. A fixed point $x$ of the second type satisfies $\operatorname{det}\left(\mathrm{id}-\mathrm{d} \phi_{x}\right)>0$.

The monotonicity of diffeomorphisms of finite type was investigated in details in the recent preprint of R. Gautschi [8]. Now we describe his results. Let $\phi$ be a diffeomorphism of finite type and $\ell$ be as in (1). Then $\phi^{\ell}$ is the product of (multiple) Dehn twists along $N$. Moreover, two parallel Dehn twists have the same sign, by (4). We say that $\phi$ has uniform twists, if $\phi^{\ell}$ is the product of only positive, or only negative Dehn twists.

Furthermore, we denote by $\ell$ the smallest positive integer such that $\phi^{\ell}$ restricts to the identity on $M \backslash N$.

If $\omega^{\prime}$ is an area form on $M$ which is the standard form $\mathrm{d} q \wedge \mathrm{d} p$ with respect to the $(q, p)$-coordinates on $N$, then $\omega:=\sum_{i=1}^{\ell}\left(\phi^{i}\right)^{*} \omega^{\prime}$ is standard on $N$ and $\phi$-invariant, i.e. $\phi \in \operatorname{Symp}(M, \omega)$. To prove that $\omega$ can be chosen such that $\phi \in \operatorname{Symp}^{m}(M, \omega)$, Gautschi distinguishes two cases: uniform and non-uniform twists. In the first case he proves the following stronger statement.

LEMMA 7 ([8]). If $\phi$ has uniform twists and $\omega$ is a $\phi$-invariant area form, then $\phi \in \operatorname{Symp}^{m}(M, \omega)$.

In the non-uniform case, monotonicity does not hold for arbitrary $\phi$-invariant area forms.

LEMMA 8 ([8]). If $\phi$ has no uniform twists, then there exists a $\phi$-invariant area form $\omega$ such that $\phi \in \operatorname{Symp}^{m}(M, \omega)$. Moreover, $\omega$ can be chosen such that it is the standard form $\mathrm{d} q \wedge \mathrm{d} p$ on $N$. 
THEOREM 9. If $\phi$ is a diffeomorphism of finite type of a compact connected surface $M$ of Euler characteristic $\chi(M)<0$ and if $\phi$ has only isolated fixed points, then $\phi$ is monotone with respect to some $\phi$-invariant area form and

$$
H F_{*}(\phi) \cong \mathbb{Z}_{2}^{N(\phi)}, \quad \operatorname{dim} H F_{*}(\phi)=N(\phi),
$$

where $N(\phi)$ denotes the Nielsen number of $\phi$.

Proof. From Lemmas 7 and 8 it follows that $\omega$ can be chosen such that $\phi \in \operatorname{Symp}^{m}(M, \omega)$. Lemma 6 implies that every $y \in \operatorname{Fix}(\phi)$ forms a different fixed point class of $\phi$, so \# $\operatorname{Fix}(\phi)=N(\phi)$. This has an immediate consequence for the Floer complex $\left(C F_{*}(\phi), \partial_{J}\right)$ with respect to a generic $J=\left(J_{t}\right)_{t \in \mathbb{R}}$. If $y^{ \pm} \in \operatorname{Fix}(\phi)$ are in different fixed point classes, then $\mathcal{M}\left(y^{-}, y^{+} ; J, \phi\right)=\emptyset$. This follows from the first equation in (2). Then the boundary map in the Floer complex is zero, $\partial_{J}=0$, and $\mathbb{Z}_{2}$-vector space $C F_{*}(\phi):=\mathbb{Z}_{2}^{\# \operatorname{Fix}(\phi)}=$ $\mathbb{Z}_{2}^{N(\phi)}$. This immediately implies $H F_{*}(\phi) \cong \mathbb{Z}_{2}^{N(\phi)}$ and $\operatorname{dim} H F_{*}(\phi)=N(\phi)$.

REMARK 10. R. Gautschi has proved in preprint [8] that, if $\phi$ is a diffeomorphism of finite type, then $\phi$ is monotone with respect to some $\phi$-invariant area form and

$$
H F_{*}(\phi) H_{*}\left(M_{\text {id }}, \partial_{M_{\text {id }}} ; \mathbb{Z}_{2}\right) \oplus \mathbb{Z}_{2}^{L\left(\phi \mid M \backslash M_{\text {id }}\right)} .
$$

Here, $L$ denotes the Lefschetz number.

In Theorem 9 the set $M_{\mathrm{id}}$ is empty and every fixed point of $\phi$ has fixed point index 1 [16]. The Lefschetz fixed point formula implies that \# $\operatorname{Fix}(\phi)=N(\phi)=L(\phi)$. So, Theorem 9 follows also from result of R. Gautschi.

\subsection{Hyperbolic diffeomorphisms of 2-dimensional torus}

THEOREM 11. If $\phi$ is a hyperbolic diffeomorphism of a 2-dimensional torus $T^{2}$, then $\phi$ is symplectic and

$$
H F_{*}(\phi) \cong \mathbb{Z}_{2}^{N(\phi)}, \quad \operatorname{dim} H F_{*}(\phi)=N(\phi),
$$

where $N(\phi)=\left|\operatorname{det}\left(E-\phi_{*}\right)\right|$ denotes the Nielsen number of $\phi$ and $\phi_{*}$ is the induced homomorphism on the fundamental group of $T^{2}$.

Proof. Hyperbolicity of $\phi$ means that the covering linear map $\tilde{\phi}: R^{2} \rightarrow R^{2}$, $\operatorname{det} \tilde{\phi}=1$, has no eigenvalues $\lambda$ with $|\lambda|=1$. The hyperbolic diffeomorphism of a 2-dimensional torus $T^{2}$ preserves the area, so it is symplectic. In fact, the covering map $\tilde{\phi}$ has a unique fixed point, which is the origin; hence, by the covering homotopy theorem, the fixed points of $\phi$ are pairwise Nielsen non-equivalent. The index of each Nielsen fixed point class, consisting of one fixed point, coincides with its Lefschetz index, and by the hyperbolicity of $\phi$, the later is not equal to zero. Thus the Nielsen number $N(\phi)=\# \operatorname{Fix}(\phi)$. It is known also that $N(\phi)=|L(\phi)|[1]$. If $y^{ \pm} \in \operatorname{Fix}(\phi)$ are in different Nielsen fixed point classes, then $\mathcal{M}\left(y^{-}, y^{+} ; J, \phi\right)=\emptyset$. This follows from the first equation in (2). Then the boundary map in the Floer complex is zero, $\partial_{J}=0$, and $\mathbb{Z}_{2}$-vector space $C F_{*}(\phi):=\mathbb{Z}_{2}^{\# \operatorname{Fix}(\phi)}=\mathbb{Z}_{2}^{N(\phi)}$. This immediately implies $H F_{*}(\phi) \cong \mathbb{Z}_{2}^{N(\phi)}$ and $\operatorname{dim} H F_{*}(\phi)=N(\phi)$.

REMARK 12. It is interesting to compare this result with the first computation by Marcin Poźniak of the Floer homology of linear symplectomorphisms in case of torus [20]. 


\section{Asymptotic invariant}

4.1. Topological entropy and the Nielsen numbers. A basic relation between Nielsen numbers and topological entropy $h(f)$ was found by N. Ivanov [12]. Here we present a very short proof of Ivanov's inequality, this proof was given by Jiang.

LEMMA $13([12])$.

$$
h(f) \geq \limsup _{n} \frac{1}{n} \cdot \log N\left(f^{n}\right) .
$$

Proof. Let $\delta$ be such that every loop in $X$ of diameter $<2 \delta$ is contractible. Let $\epsilon>0$ be a smaller number such that $d(f(x), f(y))<\delta$ whenever $d(x, y)<2 \epsilon$. Choose a point in each essential fixed point class of $f^{n}$, and let $E_{n} \subset X$ be the set consisting of these points. Thus $\left|E_{n}\right|=N\left(f^{n}\right)$. By the definition of $h(f)$, it suffices to show that $E_{n}$ is $(n, \epsilon)$-separated. Suppose the contrary. Then there would be two points $x \neq y \in E_{n}$ such that $d\left(f^{i}(x), f^{i}(y)\right) \leq \epsilon$ for $0 \leq i<n$ hence for all $i \geq 0$. Pick a path $c_{i}$ from $f^{i}(x)$ to $f^{i}(y)$ of diameter $<2 \epsilon$ for $0 \leq i<n$ and let $c_{n}=c_{0}$. By the choice of $\delta$ and $\epsilon$, $f \circ c_{i} \simeq c_{i+1}$ for all $i$, so $f^{n} \circ c_{0} \simeq c_{n}=c_{0}$. This means $x, y$ in the same fixed point class of $f^{n}$, contradicting the construction of $E_{n}$.

This inequality is remarkable in that it does not require smoothness of the map and provides a common lower bound for the topological entropy of all maps in a homotopy class.

We recall the Thurston classification theorem for homeomorphisms of surface $M$ of genus $\geq 2$.

THEOREM 14 ([23]). Every homeomorphism $\phi: M \rightarrow M$ is isotopic to a homeomorphism $f$ such that one of the following conditions is satisfied:

(1) $f$ is a periodic map;

(2) $f$ is a pseudo-Anosov map, i.e. there is a number $\lambda>1$ (stretching factor) and a pair of transverse measured foliations $\left(F^{s}, \mu^{s}\right)$ and $\left(F^{u}, \mu^{u}\right)$ such that $f\left(F^{s}, \mu^{s}\right)=\left(F^{s}, \frac{1}{\lambda} \mu^{s}\right)$ and $f\left(F^{u}, \mu^{u}\right)=\left(F^{u}, \lambda \mu^{u}\right)$;

(3) $f$ is reducible map, i.e. there is a system of disjoint simple closed curves $\gamma=$ $\left\{\gamma_{1}, \ldots, \gamma_{k}\right\}$ in the interior of $M$ such that $\gamma$ is invariant under $f$ (but $\gamma_{i}$ may be permuted) and $\gamma$ has an $f$-invariant tubular neighborhood $U$ such that each component of $M \backslash U$ has negative Euler characteristic and on each (not necessarily connected) f-component of $M \backslash U, f$ satisfies (1) or (2).

The map $f$ from Theorem 14 is called the Thurston canonical form of $f$. In (3) it can be chosen so that some iterate $f^{m}$ is a generalized Dehn twist on $U$. Such a map $f$, as well as the map $f$ in (1) or (2), will be called standard. A key observation is that if $f$ is standard, then so are all iterations of $f$.

LEMma 15 ([4]). Let $f$ be a pseudo-Anosov homeomorphism with stretching factor $\lambda>1$ of surface $M$ of genus $\geq 2$. Then

$$
h(f)=\log (\lambda)=\limsup _{n} \frac{1}{n} \cdot \log N\left(f^{n}\right) .
$$


Lemma 16 ([15]). Suppose $f$ is a standard homeomorphism of surface $M$ of genus $\geq 2$ and $\lambda$ is the largest stretching factor of the pseudo-Anosov pieces $(\lambda=1$ if there is no pseudo-Anosov pieces). Then

$$
h(f)=\log (\lambda)=\limsup _{n} \frac{1}{n} \cdot \log N\left(f^{n}\right) .
$$

The growth rate of a sequence $a_{n}$ of complex numbers is defined by

$$
\operatorname{Growth}\left(a_{n}\right):=\max \left\{1, \limsup _{n \rightarrow \infty}\left|a_{n}\right|^{1 / n}\right\}
$$

and could be equal to infinity. Note that $\operatorname{Growth}\left(a_{n}\right) \geq 1$ even if all $a_{n}=0$. When $\operatorname{Growth}\left(a_{n}\right)>1$, we say that the sequence $a_{n}$ grows exponentially.

Definition 17. We define the asymptotic invariant $F^{\infty}(g)$ of mapping class $g \in \Gamma=$ $\pi_{0}\left(\operatorname{Diff}^{+}(M)\right)$ to be the growth rate of the sequence $\left\{a_{n}=\operatorname{dim} H F_{*}\left(\phi^{n}\right)\right\}$ for a monotone representative $\phi \in \operatorname{Symp}^{m}(M, \omega)$ of $g$ :

$$
F^{\infty}(g):=\operatorname{Growth}\left(\operatorname{dim} H F_{*}\left(\phi^{n}\right)\right) .
$$

EXAMPLE 18. If $\phi$ is a non-trivial orientation preserving periodic diffeomorphism of a compact connected surface $M$ of Euler characteristic $\chi(M)<0$, then the periodicity of the sequence $\operatorname{dim} H F_{*}\left(\phi^{n}\right)$ implies that for the corresponding mapping class $g$ the asymptotic invariant

$$
F^{\infty}(g):=\operatorname{Growth}\left(\operatorname{dim} H F_{*}\left(\phi^{n}\right)\right)=1 .
$$

EXAMPLE 19. Let $\phi$ be a monotone diffeomorphism of finite type of a compact connected surface $M$ of Euler characteristic $\chi(M)<0$ and $g$ a corresponding algebraically finite mapping class. Let $U$ be the open regular neighborhood of the $k$ reducing curves $\gamma_{1}, \ldots, \gamma_{k}$ in the Thurston theorem, and $M_{j}$ be the component of $M \backslash U$. Let $F$ be a fixed point class of $\phi$. Observe that $\operatorname{ind}(F, \phi)=\operatorname{ind}\left(F, \phi_{j}\right)$ whenever $F \subset M_{j},[16]$. So if $F$ is counted in $N(\phi)$ but not counted in $\sum_{j} N\left(\phi_{j}\right)$, it must intersect $U$. But it follows from [16] that a component of $U$ can intersect at most two essential fixed point classes of $\phi$. Hence we have $N(\phi) \leq \sum_{j} N\left(\phi_{j}\right)$. For the monotone diffeomorphism of finite type $\phi$, the maps $\phi_{j}$ are periodic. Applying last inequality to $\phi^{n}$ and using Remark 10 we have

$$
\begin{aligned}
0 \leq \operatorname{dim} H F_{*}\left(\phi^{n}\right)=\operatorname{dim} & H_{*}\left(M_{\mathrm{id}}^{(n)}, \partial M_{\mathrm{id}}^{(n)} ; \mathbb{Z}_{2}\right)+N\left(\phi^{n} \mid M \backslash M_{\mathrm{id}}^{(n)}\right) \\
\leq & \operatorname{dim} H_{*}\left(M_{\mathrm{id}}^{(n)}, \partial M_{\mathrm{id}}^{(n)} ; \mathbb{Z}_{2}\right)+N\left(\phi^{n}\right) \\
& \leq \operatorname{dim} H_{*}\left(M_{\mathrm{id}}^{(n)}, \partial M_{\mathrm{id}}^{(n)} ; \mathbb{Z}_{2}\right)+\sum_{j} N\left((\phi)_{j}^{n}\right)+2 k \leq \text { Const. }
\end{aligned}
$$

by periodicity of $\phi_{j}$. Taking the growth rate in $n$, we deduce that asymptotic invariant $F^{\infty}(g)=1$.

EXAMPLE 20. Let $\phi$ be a hyperbolic automorphism of 2-dimensional torus defined by an integer matrix with eigenvalues $\lambda_{1}, \lambda_{2},\left|\lambda_{1}\right|>1$. Then $h(\phi)=\log \left(\left|\lambda_{1}\right|\right)$. On the other hand, $N\left(\phi^{n}\right)=\left|\operatorname{det}\left(I-A^{n}\right)\right|=\left|\left(1-\lambda_{1}^{n}\right)\left(1-\lambda_{2}^{n}\right)\right|$. Hence Theorem 11 implies

$$
F^{\infty}(g):=\operatorname{Growth}\left(\operatorname{dim} H F_{*}\left(\phi^{n}\right)\right)=\limsup _{n \rightarrow \infty}\left|N\left(\phi^{n}\right)\right|^{1 / n}=\exp (h(\phi))=\left|\lambda_{1}\right|>1
$$




\section{Concluding remarks and conjectures}

\subsection{Pseudo-Anosov mapping class}

ConjeCture 21. For pseudo-Anosov mapping class $g \in \Gamma=\pi_{0}\left(\operatorname{Diff}^{+}(M)\right)$ we have

$$
H F_{*}(g) \mathbb{Z}_{2}^{N(g)}, \quad \operatorname{dim} H F_{*}(g)=N(g), \quad F^{\infty}(g)=\limsup _{n \rightarrow \infty}\left|N\left(g^{n}\right)\right|^{1 / n}=h(\psi)=\lambda>1,
$$

where $N(g)$ denotes the Nielsen number of $g$ and $\psi$ is a standard (Thurston canonical form) representative of $g$, i.e. there is a monotone representative $\phi \in \operatorname{Symp}^{m}(M, \omega)$ of $g$ such that

$$
H F_{*}(\phi) \mathbb{Z}_{2}^{N(\phi)}, \quad \operatorname{dim} H F_{*}(\phi)=N(\phi), \quad F^{\infty}(g)=\limsup _{n \rightarrow \infty}\left|N\left(\phi^{n}\right)\right|^{1 / n}=h(\psi)=\lambda>1 .
$$

REMARK 22. For pseudo-Anosov "diffeomorphism" we also have, as in Theorems 4, 9 and 11 , a topological separation of fixed points $[23,16,11]$, i.e. the Nielsen number of pseudo-Anosov "diffeomorphism" equals to the number of fixed points and there are no connecting orbits between them. But we have the following difficulties in pseudo-Anosov case. Firstly, a pseudo-Anosov "diffeomorphism" is a smooth and symplectic automorphism on the complement of its fixed points set, not on the whole surface. Nevertheless, M. Gerber and A. Katok [9] have found a smooth model for pseudo-Anosov "diffeomorphism" with the same dynamical properties. More precisely, for every pseudo-Anosov "diffeomorphism" $f$ they have constructed a diffeomorphism $f^{\prime}$ that is topologically conjugate to $f$ through a homeomorphism isotopic to identity. The diffeomorphism $f^{\prime}$ is a symplectomorphism, it has the same fixed points as $f$, which are also topologically separated, and it has the same Nielsen number as $f$. Secondly, in the case of a pseudo-Anosov "diffeomorphism" and its smooth model, we have to deal with fixed points of index $-p$ where $p>1$. Such fixed points are degenerate and therefore need a local perturbation. For the proof of Conjecture 21 we need to understand the contribution of such fixed points to the Floer homology.

\subsection{The general case. Concluding remarks}

Conjecture 23. For any mapping class $g \in \Gamma=\pi_{0}\left(\operatorname{Diff}^{+}(M)\right)$ there is a monotone representative $\phi \in \operatorname{Symp}^{m}(M, \omega)$ with respect to some $\phi$-invariant area form $\omega$ such that

$$
H F_{*}(\phi) H_{*}\left(M_{\mathrm{id}}, \partial_{M_{\mathrm{id}}} ; \mathbb{Z}_{2}\right) \oplus \mathbb{Z}_{2}^{N\left(\phi \mid M \backslash M_{\mathrm{id}}\right)},
$$

where $M_{\mathrm{id}}$ denotes the union of the components of $M \backslash \operatorname{int}(U)$, where $\phi$ restricts to the identity. Let $\psi$ be a standard (Thurston canonical form) representative of $g$ and $\lambda$ is the largest stretching factor of pseudo-Anosov pieces of $\psi(\lambda:=1$ if there is no pseudo-Anosov piece). Then

$$
F^{\infty}(g):=\operatorname{Growth}\left(\operatorname{dim} H F_{*}\left(\phi^{n}\right)\right)=\lambda=h(\psi)=\limsup _{n \rightarrow \infty}\left|N\left(\psi^{n}\right)\right|^{1 / n} .
$$

REMARK 24.

(i) If $\phi \in \operatorname{Symp}^{m}(M, \omega)$ has only non-degenerate fixed points, then from the previous conjecture it follows that

$$
\# \operatorname{Fix}(\phi) \geq \operatorname{dim} H F_{*}(\phi)=\operatorname{dim} H F_{*}(\psi)=\operatorname{dim} H_{*}\left(M_{\psi=\mathrm{id}}, \partial M_{\psi=\mathrm{id}} ; \mathbb{Z}_{2}\right)+N\left(\psi \mid M \backslash M_{\psi=\mathrm{id}}\right)
$$


(ii) An analogue of the Arnold conjecture for general symplectomorphism. Together with Yuli Rudyak we propose the following conjecture: if $\phi \in \operatorname{Symp}^{m}(M, \omega)$ has only non-degenerate fixed points and there is a Hamiltonian isotopy of $\phi$ to Thurston canonical form $\psi$ then

$$
\# \operatorname{Fix}(\phi) \geq \min \{\# \operatorname{crit} f\}+\# \operatorname{Fix}\left(\psi \mid M \backslash M_{\psi=\mathrm{id}}\right)
$$

where $f$ runs over all smooth Morse functions $M_{\psi=\text { id }} \rightarrow \mathbb{R}$ such that $f>0$ on int $M_{\psi=\text { id }}$ and $f=0$ on $\partial M_{\psi=\text { id }}$ and crit $f$ is the set of critical points of $f$; if $\phi \in \operatorname{Symp}^{m}(M, \omega)$ has degenerate fixed points then

$$
\# \operatorname{Fix}(\phi) \geq \min \{\# \operatorname{crit} f\}+\# \operatorname{Fix}\left(\psi \mid M \backslash M_{\psi=\mathrm{id}}\right)
$$

where $f$ runs over all smooth functions $M_{\psi=\text { id }} \rightarrow \mathbb{R}$ such that $f>0$ on int $M_{\psi=\text { id }}$ and $f=0$ on $\partial M_{\psi=\mathrm{id}}$.

(iii) Due to P. Seidel [22] $\operatorname{dim} H F_{*}(\phi)$ is a new symplectic invariant of a four-dimensional symplectic manifold with non-zero first Betti number. This 4-manifold is produced from symplectomorphism $\phi$ by a surgery construction which is a variation of earlier constructions due to McMullen-Taubes [18]. We hope that our asymptotic invariant also give rise to a new invariant of contact 3-manifolds and symplectic 4-manifolds.

\section{References}

[1] R. Brooks, R. F. Brown, J. Pak, D. H. Taylor, Nielsen numbers of maps of tori, Proc. Amer. Math. Soc. 52 (1975), 398-400.

[2] S. Dostoglou, D. Salamon, Self dual instantons and holomorphic curves, Ann. of Math. (2) 139 (1994), 581-640.

[3] C. J. Earle, J. Eells, The diffeomorphism group of a compact Riemann surface, Bull. Amer. Math. Soc. 73 (1967), 557-559.

[4] A. Fathi, F. Laudenbach, V. Poénaru, Travaux de Thurston sur les surfaces, Astérisque $66 / 67$ (1979).

[5] A. L. Fel'shtyn, Dynamical zeta functions, Nielsen theory and Reidemeister torsion, Mem. Amer. Math. Soc. 147 (2000), no. 699.

[6] A. Floer, Morse theory for Lagrangian intersections, J. Differential Geom. 28 (1988), 513-547.

[7] A. Floer, Symplectic fixed points and holomorphic spheres, Comm. Math. Phys. 120 (1989), 575-611.

[8] R. Gautschi, Floer homology of algebraically finite mapping classes, Preprint, April 2002, math. SG/0204032.

[9] M. Gerber, A. Katok, Smooth models of Thurston's pseudo-Anosov maps, Ann. Sci. École Norm. Sup. (4) 15 (1982), 173-204.

[10] M. Gromov, Pseudoholomorphic curves in symplectic manifolds, Invent. Math. 82 (1985), 307-347.

[11] N. V. Ivanov, Nielsen numbers of mappings of surfaces, in: Studies in topology, IV, Zap. Nauchn. Sem. Leningrad. Otdel. Mat. Inst. Steklov. (LOMI) 122 (1982), 56-65.

[12] N. V. Ivanov, Entropy and Nielsen numbers, Dokl. Akad. Nauk SSSR 265 (1982), 284-287 (in Russian); English transl.: Soviet Math. Dokl. 26 (1982), 63-66. 
[13] B. Jiang, Lectures on Nielsen Fixed Point Theory, Contemp. Math. 14, Amer. Math. Soc., Providence 1983.

[14] B. Jiang, Fixed point classes from a differentiable viewpoint, in: Fixed Point Theory, Lecture Notes in Math. 886, Springer, Berlin 1981, 163-170.

[15] B. Jiang, Estimation of the number of periodic orbits, Pacific J. Math. 172 (1996), 151-185.

[16] B. Jiang, J. Guo, Fixed points of surface diffeomorphisms, Pacific J. Math. 160 (1993), $67-89$.

[17] D. McDuff, D. A. Salamon, Introduction to Symplectic Topology, Oxford Math. Monogr., Oxford Univ. Press, New York 1998.

[18] C. McMullen, C. Taubes, 4-manifolds with inequivalent symplectic forms and 3-manifolds with inequivalent fibrations, Math. Res. Lett. 6 (1999), 681-696.

[19] J. Moser, On the volume elements on a manifold, Trans. Amer. Math. Soc. 120 (1965), 286-294.

[20] M. Poźniak, Floer homology, Novikov rings and clean intersections, Northern California Symplectic Geometry Seminar, Amer. Math. Soc. Transl. Ser. 2 196, Amer. Math. Soc., Providence 1999, 119-181.

[21] P. Seidel, Symplectic Floer homology and the mapping class group, Preprint, March 2001, math. SG/0010301.

[22] P. Seidel, Braids and symplectic four-manifolds with abelian fundamental group, Preprint, February 2002, math. SG/0202135.

[23] W. P. Thurston, On the geometry and dynamics of diffeomorphisms of surfaces, Bull. Amer. Math. Soc. (N.S.) 19 (1988), 417-431. 
